https://helda.helsinki.fi

\title{
Fatal toxicity index of medicinal drugs based on a comprehensive toxicology database
}

\section{Ojanpera, llkka}

2016-09

Ojanpera , I , Kriikku , P \& Vuori , E 2016 , ' Fatal toxicity index of medicinal drugs based on a comprehensive toxicology database ' , International Journal of Legal Medicine , vol. 130 , no. 5 , pp. 1209-1216 . https://doi.org/10.1007/s00414-016-1358-8

http://hdl.handle.net/10138/225954

https://doi.org/10.1007/s00414-016-1358-8

publishedVersion

Downloaded from Helda, University of Helsinki institutional repository.

This is an electronic reprint of the original article.

This reprint may differ from the original in pagination and typographic detail.

Please cite the original version. 


\title{
Fatal toxicity index of medicinal drugs based on a comprehensive toxicology database
}

\author{
Ilkka Ojanperä $^{1} \cdot$ Pirkko Kriikku $^{1} \cdot$ Erkki Vuori $^{1}$
}

Received: 31 December 2015 / Accepted: 7 March 2016/Published online: 17 March 2016

(C) Springer-Verlag Berlin Heidelberg 2016

\begin{abstract}
The fatal toxicity index (FTI) is the absolute number of fatal poisonings caused by a particular drug divided by its consumption figure. Consequently, it is a useful measure in evaluating toxicity of the drug and its relevance in fatal poisonings. In this study, we assessed the FTI of medicinal drugs in 3 years $(2005,2009$, and 2013) in Finland. As the measure of drug consumption, we used the number of defined daily doses (DDD) per population in each year. There were 70 medicinal drugs in Finland for which the mean FTI expressed as the number of deaths per million DDD over the three study years was higher or equal to 0.1 . The Anatomical Therapeutic Chemical (ATC) classification system was used for the classification of the active ingredients of medicinal drugs according to the organ or system which they act on. Of these 70 drugs, 55 drugs $(78.6 \%$ ) acted on the nervous system (denoted by ATC code N), 11 (15.7\%) on the cardiovascular system (C), three ( $4.3 \%)$ on the alimentary tract and metabolism (A), and one $(1.4 \%)$ on the musculoskeletal system $(\mathrm{M})$. The nervous system drugs consisted of 20 psycholeptics, (ATC code N05), 20 psychoanaleptics (N06), eight analgesics (N02), six antiepileptics (N03), and one other nervous system drug (N07). The highest individual FTIs were associated with the opioids methadone, dextropropoxyphene, oxycodone, tramadol, and morphine; the antipsychotics levomepromazine and chlorprothixene; and the antidepressants doxepin, amitriptyline, trimipramine, and bupropion. Buprenorphine was not included in the study, because most of the fatal buprenorphine
\end{abstract}

Pirkko Kriikku

pirkko.kriikku@helsinki.fi

1 Department of Forensic Medicine, University of Helsinki, PO Box 40, Kytösuontie 11, FI-00014 Helsinki, Finland poisonings were due to smuggled tablets. A clearly increasing trend in FTI was observed with pregabalin and possibly with bupropion, both drugs emerging as abused substances.

Keywords FTI $\cdot$ Fatal poisonings $\cdot$ Drug sales $\cdot$ Forensic toxicology $\cdot$ Post-mortem

\section{Introduction}

Statistics on drug-related deaths are of special interest not only to clinical and forensic scientists but also to drug safety authorities and policymakers. This is due to the fact that postmortem toxicology using sophisticated analytical technology can produce more solid information on the role of illicit and prescription drugs in poisonings than is obtained from patients within the healthcare system. Comprehensive drug screening, unequivocal identification, and quantitative analysis are an essential part of the performance of post-mortem toxicology laboratories, unlike hospital laboratories that usually rely on a series of rapid immunoassay methods and thus only produce tentative identification $[1,2]$. Yet the agents involved in fatal and non-fatal poisonings are largely similar [3]. Information about the type and prevalence of drugs causing fatal poisonings are available from general registers maintained by the national central statistical offices or from special registers, such as those maintained by forensic institutions [4].

In addition to the absolute numbers of fatal poisonings caused by particular drugs, consumption figures of drugs are useful in evaluating their relevance in fatal poisonings. A practical measure of relative drug toxicity is the fatal toxicity index (FTI), which is calculated by relating the number of deaths associated with a given drug to the number of prescriptions for that drug over the same period and area [5-7]. For illicit drugs, fatal toxicity can be evaluated by relating the 
number of associated deaths to measures of availability such as seizures by law enforcement agencies [8]. Several recent studies have been published addressing the FTI for various classes of prescription drugs, such as antidepressants [9-11], sedative-hypnotic drugs [12], carisoprodol [13], and methadone $[14,15]$. Drug consumption has been expressed as the number of prescriptions, kilograms, or defined daily doses (DDD) dispensed. The DDD is the assumed average maintenance dose per day for a drug used for its main indication in adults also including the over-the-counter drugs, and thus the number of DDD is a particularly useful measure of drug consumption [16]. As fatal poisonings more often involve several drugs instead of a single substance, deaths can be classified by all drug findings, by all drugs implicated in the death, or by the most important finding [14]. In Finland, the forensic pathologist in charge of post-mortem cause-of-death investigation needs to define in the death certificate the most important drug finding of a fatal poisoning.

FTI as its many variants has proved to be a feasible tool for assessing the relative toxicity of drugs in overdose. However, the published studies have focused on specific drug categories, especially antidepressants, and have been based on various research methods within different medicolegal systems. There are no studies available aiming to establish the FTI for a multiplicity of drugs and compare the changes between years, using one comprehensive source of homogeneous data and one study setting.

Our objective was to compare the prevalence of medicinal drugs in major therapeutic categories in fatal poisonings related to their consumption in Finland between three separate years over a 9-year period: 2005, 2009, and 2013. The high post-mortem toxicology rate and comprehensive-accredited laboratory investigation produce extensive data on fatal poisonings. As a slightly different array of medicines was on sale each study year, we concentrate on those substances repeatedly implicated in fatal poisonings.

\section{Materials and methods}

The laboratory post-mortem database included a forensic pathologist's referral, laboratory analysis results, and information from the death certificate issued by a forensic pathologist. The referral contained background information from the police, such as a brief description of the circumstances of death, known medications, and the main autopsy findings. The analytical data contained analysis results for alcohols, medicines, and drugs of abuse and occasionally for other substances. Information from the final death certificate included the age and gender of the deceased, the cause of death with contributing factors according to the International Classification of Diseases (ICD-10), and the manner of death according to the World Health Organization. The manner of death in fatal poisonings was accident, suicide, or unknown. In medicinal drug poisonings, the principal drug finding indicated in the death certificate was used as the basis to classify a fatal poisoning case. Due to the structure of the death certificate, the present results are based on this most important single drug finding in each case instead of all drugs implicated in the death.

Drug consumption data, including hospital consumption, were obtained from the Finnish Medicines Agency (FIMEA), expressed as defined daily doses (DDD) per 1000 inhabitants per day (DDD/1000 inh/day). Data on fatal poisonings were obtained from the laboratory post-mortem database. The FTI was calculated by dividing the number of fatal poisonings attributed to a certain drug by the consumption of the drug over the same period. For example, there were six fatal metoprolol poisonings in Finland during 2005 (0.11 poisonings per 100,000 inhabitants), and the consumption of metoprolol in 2005, measured as the number of DDD in the whole population, was $44,274,057$. This resulted in an FTI of 0.14 deaths per million DDD for metoprolol. Only those drugs were included for which at least one fatal poisoning was recorded in at least two of the three study years. Drugs with considerable illicit trafficking were omitted from the study as the consumption of these drugs could not be assessed using the present method.

The FTI for each studied drug was calculated both for the individual study years $(2005,2009$, and 2013) and as a sum of all three years.

\section{Results}

The number of post-mortem toxicology cases investigated in Finland in years 2005, 2009, and 2013 was 6210, 6892, and 6568 , respectively, and these numbers represented 13.0, 13.8, and $12.8 \%$ of all deaths in each year, respectively. The number of fatal drug poisonings (illegal and medicinal) was 501, 636 , and 476 , respectively.

Table 1 shows the mean FTIs expressed as the number of deaths per million DDD over the three study years for those 70 medicinal drugs in the descending order for which the FTI is higher or equal to 0.1. Each fatal poisoning was categorized by the most important drug finding associated with the death as stated by the forensic pathologist in the death certificate. The Anatomical Therapeutic Chemical (ATC) classification system was used for the classification of the active ingredients of medicinal drugs according to the organ or system which they act on. Of the 70 drugs, 55 drugs $(78.6 \%)$ acted on the nervous system $(\mathrm{N}), 11(15.7 \%)$ on the cardiovascular system (C), three $(4.3 \%)$ on the alimentary tract and metabolism (A), and one $(1.4 \%)$ on the musculoskeletal system (M). The nervous system drugs consisted of 20 psycholeptics (N05), 20 
Table 1 Mean fatal toxicity index (FTI) and manner of death from 2005, 2009, and 2013 in Finland for 70 medicinal drugs according to descending order of FTI

\begin{tabular}{|c|c|c|c|c|c|c|c|}
\hline & & \multirow[t]{2}{*}{ Total deaths } & \multirow{2}{*}{$\begin{array}{l}\text { Total sales } \\
\text { DDD }\end{array}$} & \multirow{2}{*}{$\begin{array}{l}\text { Mean FTI } \\
\text { (Deaths } / 10^{6} \text { DDD) }\end{array}$} & \multicolumn{3}{|c|}{ Manner of death } \\
\hline & & & & & Suicide (\%) & Unknown (\%) & Accident $(\%)$ \\
\hline N07BC02 & Methadone & 32 & 750,333 & 42.65 & 0 & 6 & 94 \\
\hline N02AC04 & Dextropropoxyphene & 27 & 847,894 & 31.84 & 56 & 4 & 37 \\
\hline N05AA02 & Levomepromazine & 79 & $3,604,456$ & 21.92 & 48 & 15 & 37 \\
\hline N06AA12 & Doxepin & 61 & $4,359,963$ & 13.99 & 57 & 25 & 18 \\
\hline N05AF03 & Chlorprothixene & 22 & $3,095,410$ & 7.11 & 41 & 32 & 27 \\
\hline N02AA05 & Oxycodone & 51 & $7,542,630$ & 6.76 & 35 & 12 & 51 \\
\hline N06AA09 & Amitriptyline & 106 & $16,216,626$ & 6.54 & 58 & 18 & 25 \\
\hline N06AA06 & Trimipramine & 7 & $1,107,592$ & 6.32 & 43 & 14 & 43 \\
\hline N02AX02 & Tramadol & 96 & $16,886,539$ & 5.69 & 39 & 15 & 47 \\
\hline N02AA01 & Morphine & 8 & $1,527,852$ & 5.24 & 38 & 0 & 63 \\
\hline N06AX12 & Bupropion & 14 & $3,210,318$ & 4.36 & 93 & 0 & 7 \\
\hline N05AL01 & Sulpiride & 2 & 428,758 & 4.66 & 0 & 100 & 0 \\
\hline N06AA04 & Clomipramine & 6 & $1,385,030$ & 4.33 & 17 & 67 & 17 \\
\hline C07AA05 & Propranolol & 44 & $11,491,298$ & 3.83 & 91 & 5 & 5 \\
\hline N05AH04 & Quetiapine & 56 & $22,267,205$ & 2.51 & 86 & 9 & 5 \\
\hline N06AX05 & Trazodone & 2 & 820,214 & 2.44 & 100 & 0 & 0 \\
\hline N02AB03 & Fentanyl & 16 & $7,799,630$ & 2.05 & 0 & 13 & 88 \\
\hline N05AA01 & Chlorpromazine & 3 & $1,513,916$ & 1.98 & 33 & 33 & 33 \\
\hline N02AA59 & Codeine $^{\mathrm{a}}$ & 113 & $57,346,321$ & 1.97 & 38 & 20 & 42 \\
\hline N03AX16 & Pregabalin & 39 & $20,299,971$ & 1.92 & 18 & 23 & 59 \\
\hline N06AX18 & Reboxetine & 1 & 524,657 & 1.91 & 0 & 0 & 100 \\
\hline N06AB08 & Fluvoxamine & 4 & $2,277,310$ & 1.76 & 100 & 0 & 0 \\
\hline N06AA10 & Nortriptyline & 3 & $1,747,041$ & 1.72 & 33 & 33 & 33 \\
\hline N06AX03 & Mianserin & 6 & $3,718,824$ & 1.61 & 17 & 0 & 83 \\
\hline N05AH02 & Clozapine & 20 & $12,673,159$ & 1.58 & 45 & 20 & 35 \\
\hline N06AX16 & Venlafaxine & 51 & $40,567,623$ & 1.26 & 63 & 20 & 18 \\
\hline N05BA12 & Alprazolam & 41 & $40,340,908$ & 1.02 & 41 & 2 & 56 \\
\hline N06AB05 & Paroxetine & 16 & $17,126,500$ & 0.93 & 25 & 44 & 31 \\
\hline N06AG02 & Moclobemide & 3 & $3,274,090$ & 0.92 & 33 & 67 & 0 \\
\hline N03AX12 & Gabapentin & 6 & $6,579,026$ & 0.91 & 33 & 50 & 17 \\
\hline N03AF01 & Carbamazepine & 10 & $11,645,023$ & 0.86 & 50 & 30 & 20 \\
\hline N05AH03 & Olanzapine & 24 & $30,079,094$ & 0.80 & 63 & 25 & 13 \\
\hline C01AA05 & Digoxin & 20 & $27,148,383$ & 0.74 & 50 & 10 & 20 \\
\hline C01BC04 & Flecainide & 5 & $6,874,115$ & 0.73 & 80 & 0 & 20 \\
\hline N05BB01 & Hydroxyzine & 6 & $8,682,932$ & 0.69 & 50 & 17 & 33 \\
\hline C08DA01 & Verapamil & 4 & $5,806,157$ & 0.69 & 100 & 0 & 0 \\
\hline C07AB04 & Acebutolol & 3 & $4,629,967$ & 0.65 & 100 & 0 & 0 \\
\hline N06AX11 & Mirtazapine & 28 & $45,645,484$ & 0.61 & 57 & 14 & 29 \\
\hline N03AX09 & Lamotrigine & 5 & $9,036,037$ & 0.55 & 100 & 0 & 0 \\
\hline C02CA01 & Prazosin & 1 & $1,932,456$ & 0.52 & 100 & 0 & 0 \\
\hline N05CD07 & Temazepam & 45 & $87,120,115$ & 0.52 & 71 & 11 & 16 \\
\hline N05CF01 & Zopiclone & 71 & $141,258,189$ & 0.50 & 66 & 14 & 20 \\
\hline N06AX21 & Duloxetine & 5 & $10,130,365$ & 0.49 & 60 & 40 & 0 \\
\hline N03AE01 & Clonazepam & 2 & $5,531,926$ & 0.36 & 0 & 50 & 50 \\
\hline N02BA & Salicylate & 5 & $14,196,273$ & 0.35 & 40 & 40 & 20 \\
\hline N06BC01 & Caffeine & 1 & $2,840,800$ & 0.35 & 100 & 0 & 0 \\
\hline
\end{tabular}


Table 1 (continued)

\begin{tabular}{|c|c|c|c|c|c|c|c|}
\hline & & \multirow[t]{2}{*}{ Total deaths } & \multirow{2}{*}{$\begin{array}{l}\text { Total sales } \\
\text { DDD }\end{array}$} & \multirow{2}{*}{$\begin{array}{l}\text { Mean FTI } \\
\left.\text { (Deaths } / 10^{6} \mathrm{DDD}\right)\end{array}$} & \multicolumn{3}{|c|}{ Manner of death } \\
\hline & & & & & Suicide $(\%)$ & Unknown (\%) & Accident $(\%)$ \\
\hline N05CF02 & Zolpidem & 11 & $38,111,177$ & 0.29 & 82 & 0 & 18 \\
\hline M03BX02 & Tizanidine & 3 & $10,875,377$ & 0.28 & 67 & 0 & 33 \\
\hline N05AF05 & Zuclopenthixol & 1 & $3,955,418$ & 0.25 & 0 & 100 & 0 \\
\hline N05AD01 & Haloperidol & 1 & $4,074,436$ & 0.25 & 100 & 0 & 0 \\
\hline A10A & Insulins and analogs & 39 & $159,966,644$ & 0.24 & 74 & 18 & 5 \\
\hline N05AB03 & Perphenazine & 1 & $4,205,043$ & 0.24 & 100 & 0 & 0 \\
\hline C08DB01 & Diltiazem & 3 & $13,269,634$ & 0.23 & 67 & 33 & 0 \\
\hline N02BE01 & Paracetamol & 29 & $133,401,723$ & 0.22 & 34 & 21 & 41 \\
\hline N06AB04 & Citalopram and escitalopram & 31 & $150,329,960$ & 0.21 & 61 & 16 & 23 \\
\hline N03AG01 & Valproic acid & 4 & $20,031,058$ & 0.20 & 100 & 0 & 0 \\
\hline N06AB06 & Sertraline & 7 & $35,054,950$ & 0.20 & 29 & 14 & 57 \\
\hline A10BA02 & Metformin & 32 & $170,671,697$ & 0.19 & 28 & 34 & 38 \\
\hline N05BA02 & Chlordiazepoxide & 1 & $5,785,137$ & 0.17 & 0 & 100 & 0 \\
\hline N05AN & Lithium & 1 & $6,055,199$ & 0.17 & 0 & 0 & 100 \\
\hline A03FA01 & Metoclopramide & 1 & $6,103,943$ & 0.16 & 0 & 0 & 100 \\
\hline C07AA07 & Sotalol & 1 & $6,140,399$ & 0.16 & 100 & 0 & 0 \\
\hline N05CD02 & Nitrazepam & 1 & $6,183,677$ & 0.16 & 100 & 0 & 0 \\
\hline N06BA04 & Methylphenidate & 1 & $6,423,566$ & 0.16 & 0 & 100 & 0 \\
\hline N05AX08 & Risperidone & 2 & $13,623,381$ & 0.15 & 100 & 0 & 0 \\
\hline N05BA04 & Oxazepam & 6 & $41,209,268$ & 0.15 & 50 & 33 & 17 \\
\hline N06AB03 & Fluoxetine & 4 & $29,186,025$ & 0.14 & 50 & 25 & 25 \\
\hline C07AB03 & Atenolol & 3 & $24,143,785$ & 0.12 & 67 & 0 & 33 \\
\hline C07AB02 & Metoprolol & 12 & $118,750,034$ & 0.10 & 75 & 8 & 17 \\
\hline C01DA02 & Glyceryl trinitrate & 1 & $9,982,046$ & 0.10 & 100 & 0 & 0 \\
\hline
\end{tabular}

${ }^{\mathrm{a}}$ Excluding codeine combinations in cough medicines

psychoanaleptics (N06), eight analgesics (N02), six antiepileptics (N03), and one other nervous system drug (N07).

Additionally, the percentages of suicide, unknown manner of death, and accident for each drug are illustrated in Table 1.

Table 2 shows the FTIs of 56 medicinal drugs in 2005, 2009, and 2013 divided into major pharmacological categories and, within categories, arranged according to the descending order of FTI in 2013. As only those drugs were included for which at least one fatal poisoning was recorded in at least two of the three study years, a total of 40 different drugs associated with less poisoning cases were omitted from the study. Buprenorphine was not included in the study due to a high volume of illicit trafficking.

\section{Discussion}

Central nervous system drugs, especially antipsychotics (N05A), antidepressants (N06A), and opioid analgesics (N02A), comprise the majority of the drugs with a high FTI. Table 1 shows that 21 drugs have an average FTI $\geq 1.0$ deaths per million DDD based on six or more deaths over the three study years, which can be attributed to especially high toxicity. The only cardiac drug meeting these criteria is the adrenergic beta-blocking drug propranolol that, interestingly, is also prescribed for psychiatric conditions, e.g., against stage fright. As seen in Table 1, the manner of death is unknown in unexpectedly many cases, which may reflect the fact that forensic pathologists hesitate to define an overdose death as suicide without irrefutable evidence.

Among antipsychotics, our data shows that the major toxic drugs are levomepromazine, chlorprothixene, quetiapine, and clozapine. The high toxicity and the high rate of suicide among the users of the older antipsychotics are quite well known, and these factors probably explain some of our results. Unfortunately, very little data on FTIs of antipsychotic drugs is available in the literature for the comparison of our results [17]. The atypical antipsychotics quetiapine and olanzapine are to be followed carefully as there is evidence of diversion, misuse, and even dependency syndrome following a marked increase in prescribing of these agents [18]. Recently, the offlabel prescribing of quetiapine to treat anxiety and insomnia of 
Table 2 Fatal toxicity indices (FTI) in 2005, 2009, and 2013 in Finland for 56 medicinal drugs in descending order of FTI in 2013

\begin{tabular}{|c|c|c|c|c|c|c|c|c|c|c|c|c|c|}
\hline \multirow{2}{*}{\multicolumn{2}{|c|}{ ATC code }} & \multicolumn{4}{|l|}{2005} & \multicolumn{4}{|l|}{2009} & \multicolumn{4}{|l|}{2013} \\
\hline & & Deaths & $\begin{array}{l}\text { Deaths } \\
/ 10^{5} \\
\text { inhb/year }\end{array}$ & $\begin{array}{l}\text { Sales } \\
10^{6} \\
\text { DDD/ } \\
\text { year }\end{array}$ & $\begin{array}{l}\text { FTI } \\
\text { Deaths/ } \\
10^{6} \\
\text { DDD }\end{array}$ & Deaths & $\begin{array}{l}\text { Deaths } \\
/ 10^{5} \\
\text { inhb/year }\end{array}$ & $\begin{array}{l}\text { Sales } \\
10^{6} \\
\text { DDD/ } \\
\text { year }\end{array}$ & $\begin{array}{l}\text { FTI } \\
\text { Deaths/ } \\
10^{6} \\
\text { DDD }\end{array}$ & Deaths & $\begin{array}{l}\text { Deaths } \\
/ 10^{5} \\
\text { inhb/year }\end{array}$ & $\begin{array}{l}\text { Sales } \\
10^{6} \\
\text { DDD/ } \\
\text { year }\end{array}$ & $\begin{array}{l}\text { FTI } \\
\text { Deaths/ } \\
10^{6} \\
\text { DDD }\end{array}$ \\
\hline \multicolumn{14}{|c|}{ Metabolic drugs (A10) } \\
\hline A10A & Insulins and analogs & 12 & 0.23 & 41.63 & 0.29 & 12 & 0.22 & 55.90 & 0.21 & 15 & 0.28 & 62.44 & 0.24 \\
\hline A10BA02 & Metformin & 7 & 0.13 & 35.43 & 0.20 & 17 & 0.32 & 62.91 & 0.27 & 8 & 0.15 & 72.33 & 0.11 \\
\hline \multicolumn{14}{|c|}{ Cardiac drugs (C01A, C08) } \\
\hline C08DA01 & Verapamil & 1 & 0.02 & 2.38 & 0.42 & 1 & 0.02 & 1.86 & 0.54 & 2 & 0.04 & 1.57 & 1.27 \\
\hline C08DB01 & Diltiazem & 1 & 0.02 & 6.52 & 0.15 & & 0.00 & 4.14 & 0.00 & 2 & 0.04 & 2.61 & 0.77 \\
\hline C01AA05 & Digoxin & 9 & 0.17 & 11.45 & 0.79 & 6 & 0.11 & 8.67 & 0.69 & 5 & 0.09 & 7.02 & 0.71 \\
\hline C08CA01 & Amlodipine & & 0.00 & 45.18 & 0.00 & 4 & 0.07 & 68.25 & 0.06 & 9 & 0.17 & 92.34 & 0.10 \\
\hline $\mathrm{C} 01 \mathrm{BC} 04$ & Flecainide & 1 & 0.02 & 1.80 & 0.55 & 4 & 0.07 & 2.29 & 1.75 & & 0.00 & 2.79 & 0.00 \\
\hline \multicolumn{14}{|c|}{ Beta-blocking agents (C07) } \\
\hline C07AA05 & Propranolol & 14 & 0.27 & 3.66 & 3.82 & 23 & 0.43 & 3.85 & 5.98 & 7 & 0.13 & 3.98 & 1.76 \\
\hline $\mathrm{C} 07 \mathrm{AB} 02$ & Metoprolol & 6 & 0.11 & 44.27 & 0.14 & 5 & 0.09 & 40.47 & 0.12 & 1 & 0.02 & 34.00 & 0.03 \\
\hline $\mathrm{C} 07 \mathrm{AB} 07$ & Bisoprolol & 3 & 0.06 & 51.06 & 0.06 & 3 & 0.06 & 68.93 & 0.04 & 1 & 0.02 & 80.38 & 0.01 \\
\hline $\mathrm{C} 07 \mathrm{AB} 03$ & Atenolol & 1 & 0.02 & 10.90 & 0.09 & 2 & 0.04 & 7.66 & 0.26 & & 0.00 & 5.59 & 0.00 \\
\hline \multicolumn{14}{|c|}{ Antiinflammatory drugs and muscle relaxants (M01, M03) } \\
\hline M03BX02 & Tizanidine & & 0.00 & 3.20 & 0.00 & 2 & 0.04 & 3.65 & 0.55 & 1 & 0.02 & 4.02 & 0.25 \\
\hline M01AE01 & Ibuprofen & 1 & 0.02 & 78.36 & 0.01 & 1 & 0.02 & 94.89 & 0.01 & & 0.00 & 99.47 & 0.00 \\
\hline \multicolumn{14}{|c|}{ Analgesics (N02) } \\
\hline N02AA05 & Oxycodone & 9 & 0.17 & 1.84 & 4.89 & 20 & 0.37 & 2.64 & 7.58 & 22 & 0.40 & 3.06 & 7.18 \\
\hline N02AX02 & Tramadol & 21 & 0.40 & 5.37 & 3.91 & 35 & 0.65 & 5.61 & 6.24 & 40 & 0.73 & 5.91 & 6.77 \\
\hline N02AB03 & Fentanyl & & 0.00 & 2.92 & 0.00 & 6 & 0.11 & 2.72 & 2.21 & 10 & 0.18 & 2.17 & 4.61 \\
\hline N02AA59 & Codeine $^{\mathrm{a}}$ & 45 & 0.86 & 18.17 & 2.48 & 46 & 0.86 & 20.12 & 2.29 & 22 & 0.40 & 19.06 & 1.15 \\
\hline N02BE01 & Paracetamol & 5 & 0.10 & 28.97 & 0.17 & 7 & 0.13 & 46.10 & 0.15 & 17 & 0.31 & 58.34 & 0.29 \\
\hline N02AA01 & Morphine & 1 & 0.02 & 0.38 & 2.61 & 7 & 0.13 & 0.53 & 13.27 & & 0.00 & 0.62 & 0.00 \\
\hline N02BA & Salicylate & 1 & 0.02 & & & 4 & 0.07 & 9.08 & 0.44 & & 0.00 & 5.11 & 0.00 \\
\hline N02AC04 & Dextropropoxyphene & 13 & 0.25 & 0.63 & 20.54 & 13 & 0.24 & 0.21 & 60.50 & 1 & 0.02 & 0.00 & \\
\hline \multicolumn{14}{|c|}{ Antiepileptics (N03) } \\
\hline N03AX16 & Pregabalin & 1 & 0.02 & 1.84 & 0.54 & 12 & 0.22 & 7.79 & 1.54 & 26 & 0.48 & 10.66 & 2.44 \\
\hline N03AF01 & Carbamazepine & 6 & 0.11 & 4.35 & 1.38 & 3 & 0.06 & 3.83 & 0.78 & 1 & 0.02 & 3.46 & 0.29 \\
\hline N03AG01 & Valproic acid & 1 & 0.02 & 5.81 & 0.17 & 2 & 0.04 & 6.82 & 0.29 & 1 & 0.02 & 7.40 & 0.14 \\
\hline N03AX09 & Lamotrigine & 2 & 0.04 & 2.07 & 0.97 & 3 & 0.06 & 3.18 & 0.94 & & 0.00 & 3.78 & 0.00 \\
\hline N03AX12 & Gabapentin & 1 & 0.02 & 1.92 & 0.52 & 5 & 0.09 & 1.88 & 2.67 & & 0.00 & 2.79 & 0.00 \\
\hline \multicolumn{14}{|c|}{ Antipsychotics (N05A) } \\
\hline N05AA02 & Levomepromazine & 38 & 0.72 & 1.46 & 26.06 & 26 & 0.49 & 1.19 & 21.82 & 15 & 0.28 & 0.96 & 15.71 \\
\hline N05AF03 & Chlorprothixene & 11 & 0.21 & 1.32 & 8.31 & 3 & 0.06 & 1.02 & 2.95 & 8 & 0.15 & 0.76 & 10.58 \\
\hline N05AL01 & Sulpiride & 1 & 0.02 & 0.17 & 5.79 & & 0.00 & 0.14 & 0.00 & 1 & 0.02 & 0.12 & 8.38 \\
\hline N05AH04 & Quetiapine & 11 & 0.21 & 4.01 & 2.74 & 25 & 0.47 & 7.87 & 3.18 & 20 & 0.37 & 10.39 & 1.93 \\
\hline N05AH02 & Clozapine & 5 & 0.10 & 3.74 & 1.34 & 7 & 0.13 & 4.34 & 1.61 & 8 & 0.15 & 4.60 & 1.74 \\
\hline N05AH03 & Olanzapine & 7 & 0.13 & 8.29 & 0.84 & 9 & 0.17 & 10.41 & 0.86 & 8 & 0.15 & 11.38 & 0.70 \\
\hline N05AA01 & Chlorpromazine & 2 & 0.04 & 0.75 & 2.67 & 1 & 0.02 & 0.55 & 1.83 & & 0.00 & 0.22 & 0.00 \\
\hline \multicolumn{14}{|c|}{ Anxiolytics (N05B) } \\
\hline N05BB01 & Hydroxyzine & & 0.00 & 2.67 & 0.00 & 3 & 0.06 & 2.79 & 1.07 & 3 & 0.06 & 3.22 & 0.93 \\
\hline N05BA12 & Alprazolam & 13 & 0.25 & 14.52 & 0.90 & 19 & 0.36 & 14.24 & 1.33 & 9 & 0.17 & 11.58 & 0.78 \\
\hline N05BA04 & Oxazepam & 1 & 0.02 & 14.75 & 0.07 & & 0.00 & 14.16 & 0.00 & 5 & 0.09 & 12.30 & 0.41 \\
\hline
\end{tabular}

Hypnotics and sedatives (N05C) 
Table 2 (continued)

\begin{tabular}{|c|c|c|c|c|c|c|c|c|c|c|c|c|c|}
\hline \multirow[t]{2}{*}{ ATC code } & & \multicolumn{4}{|l|}{2005} & \multicolumn{4}{|l|}{2009} & \multicolumn{4}{|l|}{2013} \\
\hline & & Deaths & $\begin{array}{l}\text { Deaths } \\
/ 10^{5} \\
\text { inhb/year }\end{array}$ & $\begin{array}{l}\text { Sales } \\
10^{6} \\
\text { DDD/ } \\
\text { year }\end{array}$ & $\begin{array}{l}\text { FTI } \\
\text { Deaths/ } \\
10^{6} \\
\text { DDD }\end{array}$ & Deaths & $\begin{array}{l}\text { Deaths } \\
/ 10^{5} \\
\text { inhb/year }\end{array}$ & $\begin{array}{l}\text { Sales } \\
10^{6} \\
\text { DDD/ } \\
\text { year }\end{array}$ & $\begin{array}{l}\text { FTI } \\
\text { Deaths/ } \\
10^{6} \\
\text { DDD }\end{array}$ & Deaths & $\begin{array}{l}\text { Deaths } \\
/ 10^{5} \\
\text { inhb/year }\end{array}$ & $\begin{array}{l}\text { Sales } \\
10^{6} \\
\text { DDD/ } \\
\text { year }\end{array}$ & $\begin{array}{l}\text { FTI } \\
\text { Deaths/ } \\
10^{6} \\
\text { DDD }\end{array}$ \\
\hline N05CD07 & Temazepam & 13 & 0.25 & 35.39 & 0.37 & 19 & 0.36 & 30.12 & 0.63 & 13 & 0.24 & 21.61 & 0.60 \\
\hline N05CF01 & Zopiclone & 30 & 0.57 & 51.54 & 0.58 & 29 & 0.54 & 49.18 & 0.59 & 12 & 0.22 & 40.53 & 0.30 \\
\hline N05CF02 & Zolpidem & 4 & 0.08 & 11.72 & 0.34 & 4 & 0.07 & 13.50 & 0.30 & 3 & 0.06 & 12.89 & 0.23 \\
\hline \multicolumn{14}{|c|}{ Antidepressants (N06A) } \\
\hline N06AA12 & Doxepin & 22 & 0.42 & 1.84 & 11.95 & 30 & 0.56 & 1.52 & 19.69 & 9 & 0.17 & 0.99 & 9.05 \\
\hline N06AX12 & Bupropion & 1 & 0.02 & 0.81 & 1.24 & & 0.00 & 0.16 & 0.00 & 13 & 0.24 & 2.25 & 5.78 \\
\hline N06AA06 & Trimipramine & 4 & 0.08 & 0.56 & 7.19 & 2 & 0.04 & 0.31 & 6.40 & 1 & 0.02 & 0.24 & 4.19 \\
\hline N06AA09 & Amitriptyline & 46 & 0.88 & 4.74 & 9.71 & 39 & 0.73 & 5.45 & 7.16 & 21 & 0.39 & 6.03 & 3.48 \\
\hline N06AX16 & Venlafaxine & 11 & 0.21 & 8.15 & 1.35 & 24 & 0.45 & 13.73 & 1.75 & 16 & 0.29 & 18.68 & 0.86 \\
\hline N06AB05 & Paroxetine & 6 & 0.11 & 6.37 & 0.94 & 6 & 0.11 & 5.68 & 1.06 & 4 & 0.07 & 5.07 & 0.79 \\
\hline N06AX21 & Duloxetine & & 0.00 & 0.23 & 0.00 & 1 & 0.02 & 4.73 & 0.21 & 4 & 0.07 & 5.17 & 0.77 \\
\hline N06AX11 & Mirtazapine & 9 & 0.17 & 12.10 & 0.74 & 16 & 0.30 & 16.29 & 0.98 & 3 & 0.06 & 17.25 & 0.17 \\
\hline N06AB06 & Sertraline & 3 & 0.06 & 9.69 & 0.31 & 2 & 0.04 & 11.80 & 0.17 & 2 & 0.04 & 13.57 & 0.15 \\
\hline N06AB04 & $\begin{array}{r}\text { Citalopram and } \\
\text { escitalopram }\end{array}$ & 10 & 0.19 & 40.02 & 0.25 & 14 & 0.26 & 55.20 & 0.25 & 7 & 0.13 & 55.12 & 0.13 \\
\hline N06AB03 & Fluoxetine & & 0.00 & 10.65 & 0.00 & 3 & 0.06 & 9.84 & 0.30 & 1 & 0.02 & 8.70 & 0.12 \\
\hline N06AA04 & Clomipramine & 3 & 0.06 & 0.52 & 5.79 & 3 & 0.06 & 0.45 & 6.68 & & 0.00 & 0.42 & 0.00 \\
\hline N06AA10 & Nortriptyline & 1 & 0.02 & 0.33 & 3.07 & 2 & 0.04 & 0.63 & 3.20 & & 0.00 & 0.80 & 0.00 \\
\hline N06AB08 & Fluvoxamine & 1 & 0.02 & 0.96 & 1.04 & 3 & 0.06 & 0.80 & 3.75 & & 0.00 & 0.52 & 0.00 \\
\hline N06AG02 & Moclobemide & 2 & 0.04 & 1.29 & 1.56 & 1 & 0.02 & 1.11 & 0.90 & & 0.00 & 0.88 & 0.00 \\
\hline N06AX03 & Mianserin & 3 & 0.06 & 1.57 & 1.91 & 3 & 0.06 & 1.23 & 2.44 & & 0.00 & 0.92 & 0.00 \\
\hline \multicolumn{14}{|c|}{ Drugs used in addictive disorders (N07B) } \\
\hline N07BC02 & Methadone & 1 & 0.02 & 0.10 & 10.43 & 20 & 0.37 & 0.12 & 170.65 & 11 & 0.20 & 0.54 & 20.48 \\
\hline
\end{tabular}

${ }^{a}$ Excluding codeine combinations in cough medicines

drug addicts has been increasing markedly in Finland and other countries [19].

Among antidepressants, the highest relative toxicity based on our data is seen with doxepin, amitriptyline, trimipramine, bupropion, clomipramine, mianserin, and venlafaxine. The present study confirms the earlier findings that the FTIs for the tricyclic antidepressants are significantly higher than those for the selective serotonin reuptake inhibitors (SSRI), while the other antidepressants fall in between [20-22]). Swedish researchers reported FTI values based on DDD but considered only fatal mono-intoxications due to one single drug in their calculations, and consequently their FTI values were lower than here [9]. Bupropion, a cathinone derivative, is currently prescribed both for smoking cessation and depression. As indicated in Table 2, the FTI of bupropion is drastically higher in 2013 compared to the two earlier study years. There are recent reports in the literature concerning bupropion abuse [23], but it is not yet known if the increase of FTI in our material is due to emerging bupropion abuse or other reasons.
Among opioid analgesics, methadone, dextropropoxyphene, oxycodone, tramadol, morphine, fentanyl, and codeine are the drugs with the highest FTI. The strong opioids methadone, oxycodone, morphine, and fentanyl involve much abuse resulting in unintentional deaths, while the manner of death for the weak opioids dextropropoxyphene, tramadol, and codeine is often more difficult to establish. Buprenorphine, one of the most important drugs that had caused fatal poisonings in Finland, is not included in the present study, because most of the fatal buprenorphine poisonings were due to smuggled tablets [24]. Methadone is predominantly used in opioid maintenance treatment, and the drug is associated with a substantial amount of abuse and diversion. However, the insignificance of its illicit trafficking allows us to include methadone in this study. The FTI of methadone, although showing large variation between the study years, compares well with the essentially similar OD4 score reported to be $20-63$ in Victoria, Australia, during 1990-2005 [15]. Dextropropoxyphene is one of the few drugs that have been recalled from the market due to abuse and 
excessive overdose deaths - only to be replaced by another problematic drug, tramadol.

Two sedative drugs, pregabalin and alprazolam, stand out in our study by having caused fatal poisonings especially in combination with opioids. Pregabalin is used in the treatment of neuropathic pain and epilepsy, as well as in generalized anxiety disorder. The drug has a steadily increasing FTI during the study years. This is obviously due to the fact that pregabalin with high doses produces benzodiazepine-like effects and thus possesses considerable abuse potential as such or as an opioid booster [25]. Alprazolam is the benzodiazepine with a high abuse liability [26], and it shows the highest FTI of all benzodiazepines in our material. Swedish researchers published FTI values for sedative and hypnotic drugs based either on fatal mono-intoxications or multi-drug intoxications [12]. The former values were understandably always lower than in our study but the latter were fairly similar to ours, e.g., for zopiclone (0.79), zolpidem (0.52), and alprazolam (0.52).

The changes observed in the number of fatal intoxications caused by a certain drug in the study period mainly followed the changes in the sales of the drug (Table 2). It could be hypothesized that if the FTI for an individual drug remained similar between the years, the result would represent an accurate measure of the relative toxicity of this substance. Our data roughly supports this hypothesis as the typical antipsychotics, tricyclic antidepressants, and opioids rank steadily high, followed by atypical antipsychotics and other antidepressants, while sedative-hypnotics, anxiolytics, antiepileptics, and SSRI drugs had somewhat lower FTIs. However, prescription practices, abuse potential, and the patient's perception of toxicity associated with a drug play a major role, and the FTI may change in time as can be seen with pregabalin and possibly with bupropion.

There are some limitations in this study. Most fatal poisonings are caused by multiple drugs taken simultaneously, while the present results are based on the most important single drug finding in each case. The contribution of other drugs present in these poisoning cases could not be taken into account. Occasionally, it can be challenging for the forensic pathologist to point out a single substance as the underlying cause of death. The number of cases attributed to certain drugs listed in the tables is too low to draw statistically significant conclusions. The fatal poisonings due to citalopram and escitalopram could not be differentiated in the present material. Finally, the sales figures of cough medicines (syrups) containing codeine were not available to us.

\section{Conclusions}

Instead of simply calculating the absolute numbers of poisoning deaths, the FTI measures relative toxicity and ranks various medicinal drugs according to their lethality. This information helps forensic toxicologists and pathologists in interpreting drug findings in the cause-of-death investigation. In addition, policymakers can exploit the FTI in monitoring the lethality of medicinal drugs in time and detect possible trends that require intervention. The number of DDD is a particularly useful measure of drug consumption, and these figures are usually readily available. The present study has determined the FTI for a larger set of different drugs than has been reported earlier.

Compliance with ethical standards The study was conducted in accordance with all applicable local and international laws and regulations. For this type of study, formal consent is not required.

Conflict of interest The authors declare that they have no conflict of interest.

\section{References}

1. von Mach MA, Weber C, Meyer MR, Weilemann LS, Maurer HH, Peters FT (2007) Comparison of urinary on-site immunoassay screening and gas chromatography-mass spectrometry results of 111 patients with suspected poisoning presenting at an emergency department. Ther Drug Monit 29:27-39

2. Sundström M, Pelander A, Ojanperä I (2014) Comparison between drug screening by immunoassay and ultra-high performance liquid chromatography/high-resolution time-of-flight mass spectrometry in post-mortem urine. Drug Test Anal 7:420-427

3. Pohjola-Sintonen S, Kivistö KT, Vuori E, Lapatto-Reiniluoto O, Tiula E, Neuvonen PJ (2000) Identification of drugs ingested in acute poisoning: correlation of patient history with drug analyses. Ther Drug Monit 22:749-752

4. Handley SA, Flanagan RJ (2014) Drugs and other chemicals involved in fatal poisoning in England and Wales during 2000-2011. Clin Toxicol 52:1-12

5. Girdwood RH (1974) Death after taking medicaments. Br Med J 1: 501-504

6. King LA, Moffat AC (1981) Hypnotics and sedatives: an index of fatal toxicity. Lancet 14:387-388

7. King LA, Moffat AC (1983) A possible index of fatal drug toxicity in humans. Med Sci Law 23:193-198

8. King LA, Corkery JM (2010) An index of fatal toxicity for drugs of misuse. Hum Psychopharmacol 25:162-166

9. Reis M, Aamo T, Ahlner J, Druid H (2007) Reference concentrations of antidepressants. A compilation of postmortem and therapeutic levels. J Anal Toxicol 31:254-264

10. Hawton K, Bergen H, Simkin S, Cooper J, Waters K, Gunnell D, Kapur N (2010) Toxicity of antidepressants: rates of suicide relative to prescribing and non-fatal overdose. Br J Psychiatry 196:354-358

11. Launiainen T, Rasanen I, Vuori E, Ojanperä I (2011) Fatal venlafaxine poisonings are associated with a high prevalence of drug interactions. Int J Legal Med 125:349-358

12. Jönsson AK, Söderberg C, Espnes KA, Ahlner J, Eriksson A, Reis M, Druid H (2014) Sedative and hypnotic drugs - fatal and nonfatal reference blood concentrations. Forensic Sci Int 236:138-145

13. Høiseth G, Bramness JG, Christophersen AS, Mørland J (2007) Carisoprodol intoxications: a retrospective study of forensic autopsy material from 1992-2003. Int J Legal Med 121:403-409 
14. Strang J, Hall W, Hickman M, Bird SM (2010) Impact of supervision of methadone consumption on deaths related to methadone overdose (1993-2008): analyses using OD4 index in England and Scotland. BMJ 341:c4851

15. Pilgrim JL, McDonough M, Drummer OH (2013) A review of methadone deaths between 2001 and 2005 in Victoria, Australia. Forensic Sci Int 226:216-222

16. Vuori E, Ruohonen A, Penttilä A, Klaukka T, Lahti T (1989) Fatal poisonings with antidepressants in Finland 1985-1987. Acta Psychiatr Scand Suppl 354:55-60

17. Griffiths C, Flanagan RJ (2005) Fatal poisoning with antipsychotic drugs, England and Wales 1993-2002. J Psychopharmacol 19:667674

18. Montebello ME, Brett J (2015) Misuse and associated harms of quetiapine and other atypical antipsychotics. In: Current Topics in Behavioral Neurosciences, Springer International Publishing Switzerland 2015, pp 1-15

19. Carton L, Cottencin O, Lapeyre-Mestre M, Geoffroy PA, Favre J, Simon N, Bordet R, Rolland B (2015) Off-label prescribing of antipsychotics in adults, children and elderly individuals: a systematic review of recent prescription trends. Curr Pharm Des 21:32803297
20. Cassidy S, Henry J (1987) Fatal toxicity of antidepressant drugs in overdose. Br Med J (Clin Res Ed) 295:1021-1024. Erratum in: Br Med J (Clin Res Ed) 295:1382

21. Buckley NA, McManus PR (2002) Fatal toxicity of serotoninergic and other antidepressant drugs: analysis of United Kingdom mortality data. BMJ 325:1332-1333

22. Koski A, Vuori E, Ojanperä I (2005) Newer antidepressants: evaluation of fatal toxicity index and interaction with alcohol based on Finnish postmortem data. Int J Legal Med 119:344-348

23. Oppek K, Koller G, Zwergal A, Pogarell O (2014) Intravenous administration and abuse of bupropion: a case report and a review of the literature. J Addict Med 8:290-293

24. Häkkinen M, Heikman P, Ojanperä I (2013) Parenteral buprenorphine-naloxone abuse is a major cause of fatal buprenorphine-related poisoning. Forensic Sci Int 232:11-15

25. Häkkinen M, Vuori E, Kalso E, Gergov M, Ojanperä I (2014) Profiles of pregabalin and gabapentin abuse by postmortem toxicology. Forensic Sci Int 241:1-6

26. Shah NA, Abate MA, Smith MJ, Kaplan JA, Kraner JC, Clay DJ (2012) Characteristics of alprazolam-related deaths compiled by a centralized state medical examiner. Am J Addict 21:S27-34 